\title{
Estimativa de área impermeabilizada diretamente conectada e sua utilização como coeficiente de escoamento superficial
}

\section{Estimate of directly connected impervious areas and its use as runoff coefficient}

\author{
Leonardo Monteiro Garotti \\ Engenheiro Civil pela Universidade Federal de São Carlos (UFSCar). Mestre em Engenharia Urbana pela UFSCar
}

Ademir Paceli Barbassa

Engenheiro Civil pela Universidade Federal de Minas Gerais (UFMG). Doutor em Hidráulica e Saneamento pela Universidade de São Paulo (USP). Pósdoutor pela Universidade do Colorado, Boulder, Estados Unidos. Professor-associado do Departamento de Engenharia Civil (DECiv) e do Programa de

Pós-Graduação em Engenharia Urbana da UFSCar

\section{Resumo}

Neste estudo realizou-se a caracterização do uso e ocupação do solo na cidade de Ribeirão Preto (SP), além da análise detalhada da impermeabilização urbana e conectividade hidráulica dos lotes. Também foi avaliado o coeficiente de escoamento superficial (C) para seis sub-bacias, utilizando-se três métodos com diferentes níveis de precisão. Utilizando-se o Método Racional com os valores estimados de C, calcularam-se os picos de vazão para as sub-bacias. Identificou-se que a impermeabilização cresce com a redução da área do lote. As estimativas de $\mathrm{C}$ resultam em diferenças importantes em seus valores refletindo na previsão da vazão. A utilização de tabelas com $\mathrm{C}$ detalhado e no seu limite superior se aproxima dos valores estimados por $\mathrm{C}_{\text {AIDC. }}$ Recomenda-se estimar $\mathrm{C}_{\mathrm{AIDC}}$ a partir da área do lote ou pesquisa de campo.

Palavras-chave: coeficiente de escoamento superficial, drenagem urbana, área impermeabilizada diretamente conectada.

\begin{abstract}
This paper presents a study of characterization of land occupation and its use in the city of Ribeirão Preto (São Paulo), as well as a detailed analysis of the urban imperviousness and hydraulic connectivity of the plots. The runoff coefficient (C) for siz sub-basins was evaluated on the basis of three different definitions. The peaks of outflow for the sub-basins were calculated using the Rational Method with the estimated values of $\mathrm{C}$. It was identified that the imperviousness increases as the area of the plot decreases. The estimative of the $\mathrm{C}$ show important differences in its values, influencing the outflow. The use of tables with detailed and maximal $\mathrm{C}$ values beyond its superior limitation is equivalent to the values estimated with $\mathrm{C}_{\mathrm{DCIA}}$. It is recommended that the $\mathrm{C}_{\mathrm{DCIA}}$ relative to the area be estimated based on the plot or field researches.
\end{abstract}

Keywords: runoff coefficient, urban draining, directly connected impervious areas.

\section{Introdução e objetivos}

A caracterização urbana, do ponto de vista hidrológico, envolve um grande número de variáveis, dependendo do modelo que se quer aplicar. As dificuldades estão na falta de registros hidrológicos, levantamentos topográficos incompletos, precários ou defasados e informações cadastrais inexistentes.

Dessa forma, mesmo modelos simplificados como o método racional têm seus parâmetros de entrada adotados mais pela experiência do projetista do que pautados por um método que assegure confiança nos valores obtidos.
O conhecimento da superfície urbana é elemento essencial também para o planejamento urbanístico, estabelecimento de legislações próprias, etc. Alguns trabalhos têm sido feitos com este intuito, aumentando o leque de métodos. Entretanto, há muito a conhecer sobre a dinâmica de vários parâmetros importantes, entre eles a impermeabilização do solo, que influencia diretamente no coeficiente de escoamento superficial.

O coeficiente de escoamento superficial de uma bacia geralmente envolve estimativas de áreas com características homogêneas e seus respectivos coeficientes de escoamento superficial, para que, ponderadamente, seja possível calculá-lo para a bacia em estudo. Estas duas 
estimativas podem ser realizadas por diversos métodos, e aí reside o interesse e foco deste trabalho.

Neste estudo, além da caracterização do uso e ocupação do solo e das características de impermeabilização na cidade de Ribeirão Preto (SP), utilizando-se de dados cadastrais da Prefeitura municipal e visitas in loco, empregaram-se as áreas impermeabilizadas diretamente conectadas como base para estimativa do coeficiente de escoamento superficial e comparação com outros métodos.

\section{Impermeabilização do solo}

O método racional se caracteriza pela simplicidade dos mecanismos numéricos de quantificação utilizados, onde todos os processos hidrológicos para transformação da precipitação em vazão são englobados em apenas um coeficiente, o coeficiente de escoamento superficial (C). Este método é amplamente utilizado, tendo se tornado uma regra no dimensionamento de pequenos sistemas de drenagem pluvial. São consideradas as hipóteses: a chuva uniformemente distribuída no tempo e no espaço; o período de retorno da precipitação é igual ao da vazão de pico; o C é constante durante a chuva e de chuva para chuva e é estimado principalmente de acordo com as características da bacia; a duração da precipitação intensa de projeto é igual ao tempo de concentração. Não são avaliados o volume de cheias e a distribuição temporal das vazões. Dessa forma, a bacia deve ser suficientemente pequena para que tal situação seja admitida, geralmente restrita a áreas de até $2 \mathrm{~km}^{2}$

O coeficiente de escoamento superficial, relação entre as alturas efetiva e precipitada, é baseado em uma série de fatores, como as características da superfície, o tipo de solo, a umidade antecedente, a permeabilidade do solo, a ocupação da bacia, o tempo de concentração. Usualmente, o coeficiente de escoamento é adotado em função de características de urbanização, como mostra a Tabela 1, ou de características detalhadas das diversas superfícies encontradas na bacia, conforme a Tabela 2 .

A impermeabilização do solo é um importante parâmetro urbanístico que reflete o impacto da urbanização sobre o sistema de drenagem de águas pluviais, podendo ser utilizado na estimativa de valores apropriados para $C$.

De acordo com Booth e Jackson (1997), apesar de largamente utilizado e aceito na caracterização dos impactos causados pela urbanização sobre a hidrologia urbana, o conceito de área impermeabilizada total (AIT) apresenta algumas questões não resolvidas. Entre elas, a mais relevante é a distinção entre as áreas impermeabilizada total e efetiva. A AIT representa a porcentagem total de área impermeabilizada na bacia, ou seja, a quantidade de superfícies cobertas por edificações ou pavimentos que impeçam a passagem de água para o subsolo. Já a área impermeabilizada efetiva é definida como a área impermeabilizada diretamente conectada (AIDC) ao sistema de drenagem urbana, ou seja, áreas que contribuem efetivamente para o escoamento superficial direto, não passando por áreas permeáveis, nem tendo oportunidade de infiltração no solo.

Segundo Lee e Heaney (2003), o coeficiente de escoamento superficial deve ser proporcional à área impermeabilizada. Segundo esses autores, na versão original britânica do Método Racional, utilizava-se como coeficiente de escoamento a porcentagem de área impermeabilizada diretamente conectada, ou seja, era considerado 100\% o escoamento provindo dessas áreas.

Porém, as taxas de impermeabilização e suas características específicas são difíceis de serem medidas com precisão e muitas têm sido as metodologias adotadas para quantificação e estimativa deste parâmetro. Dentre elas, pode-se citar a relação da impermeabilização em função de outros parâmetros urbanos, como a densidade demográfica e a taxa de ocupação; ou medidas diretas em fotografias aéreas, imagens de satélite e medidas de campo.

Lee e Heaney (2003) realizaram um estudo sobre estimativa de impermeabilização e análise de seus impactos no sistema de drenagem urbana. A quantificação das áreas impermeabilizadas e impermeabilizadas diretamente conectadas foi detalhada em cinco níveis de exatidão, desde dados provindos de relações obtidas na bibliografia até a utilização de geoprocessamento, fotografias aéreas e visitas de campo. Nas visitas de campo, foram verificadas a conectividade hidráulica e as condições de cada superfície impermeabilizada, inclusive as estruturas de descargas de águas pluviais provenientes dos telhados. Esse detalhamento foi aplicado a uma área residencial de 5,81 hectares em Boulder, Colorado, EUA. Os resultados para esta área de estudo mostraram área impermeabilizada total (AIT) de 35,9\% e AIDC de 13\%. Observou-se, ainda, que 97,2\% da AIDC estava sendo usada por área destinada ao transporte. A modelagem hidrológica desta área mostra uma diferença nas vazões de pico da ordem de $265 \%$, de acordo com a impermeabilização medida nos diferentes níveis de detalhamento. Segundo os autores, esses resultados sugerem a necessidade de focar as AIDC como o principal indicador dos efeitos da urbanização sobre a qualidade e aumento do escoamento superficial.

Boyd, Bufill e Knee (1993; 1994) analisaram numerosos dados de chuvas e escoamento superficial em 26 bacias urbanas localizadas em 12 países com o intuito de prever o escoamento superficial proveniente de áreas permeáveis e impermeabilizadas. Foram consideradas duas séries de dados: com o escoamento superficial proveniente somente de áreas impermeabilizadas para chuvas de baixa intensidade; e escoamento superficial proveniente tanto de áreas impermeabilizadas quanto permeáveis, porém para chuvas mais intensas. Segundo os autores, através dos cruzamentos dos dados de chuva e escoamento superficial é possível identificar a área impermeabilizada diretamente 
conectada (AIDC) utilizando as equações empíricas 1 e 2. Os resultados dos cruzamentos apresentaram bons ajustes, com $\mathrm{R}^{2}$ em torno de 0,85, porém a AIDC foi estimada por meio de mapas das bacias estudadas.

$$
\begin{aligned}
& F_{i}=0,75 * A_{i} / A \\
& F_{i}=0,87 * A_{i c} / A
\end{aligned}
$$

onde:

$F_{i}$ : fração impermeabilizada efetiva;

$A_{i}$ : área impermeabilizada total;

A : área total;

$A_{i c}$ : área impermeabilizada diretamente conectada (AIDC).

As áreas impermeabilizadas diretamente conectadas têm sido detalhadas e consideradas em alguns estudos, entre eles: Boyd, Bufill e Knee (1993; 1994); Lee e Heaney (2003); Fontes e Barbassa (2001, 2003), Garotti e Barbassa (2005); Garotti, Imoto e Barbassa (2007). Esses estudos forneceram importantes relações com outros parâmetros urbanos de fácil acesso, tais como taxa de ocupação, área de lote, entre outros, o que facilita a estimativa e utilização da AIDC, no processo de planejamento urbano.

\section{Método para caracterização urbanística}

Os elementos espaciais estudados foram os lotes urbanos. Segundo o cadastro técnico fornecido pela Secretaria Municipal de Planejamento de Ribeirão Preto (SMPRP, 2004a; 2004b), a cidade contava com 184.847 lotes, sendo 125.445 ocupados por edificações de qualquer natureza, o restante seriam lotes vagos. Devido à grande variabilidade e número de lotes, foi preciso realizar um estudo estatístico para determinação da quantidade de amostras a serem coletadas, visando estimativas e análises satisfatórias dos parâmetros. A caracterização foi realizada levando-se em consideração os lotes ocupados, partindo-se do princípio que os lotes vagos apresentam as mesmas características de uso, ocupação e impermeabilização.

No entanto, para caracterização das sub-bacias, as áreas referentes aos lotes vagos e arruamentos foram quantificadas. Para esta análise, os lotes vagos foram considerados como área 100\% permeável, e as áreas referentes ao arruamento foram consideradas 100\% impermeabilizadas.

A caracterização foi realizada através da separação dos lotes urbanos em estratos, de acordo com o uso do solo e tamanho do lote no caso de lotes residenciais. A metodologia utilizada para realizar esta separação está apresentada no item a seguir. Dessa forma, foi possível verificar a representatividade de área e o número de lotes para cada estrato proposto, além da investigação in loco e posterior análise dos seguintes parâmetros:
Tabela 1 - Valores de C, conforme as características de urbanização

\begin{tabular}{|c|c|}
\hline Zonas & Valores de C \\
\hline $\begin{array}{l}\text { De edificação muito densa: partes centrais densamente } \\
\text { construídas de uma cidade com ruas e calçadas pavi- } \\
\text { mentadas; }\end{array}$ & 0,70 a 0,95 \\
\hline $\begin{array}{l}\text { De edificação não muito densa: partes adjacentes ao } \\
\text { centro, de menor densidade de habitações, mas com } \\
\text { ruas e calçadas pavimentadas; }\end{array}$ & 0,60 a 0,70 \\
\hline $\begin{array}{l}\text { De edificação com pouca superfície livre: partes residen- } \\
\text { ciais com construções cerradas, ruas pavimentadas; }\end{array}$ & 0,50 a 0,60 \\
\hline $\begin{array}{l}\text { De edificação com muitas superfícies livres: partes } \\
\text { residenciais tipo cidade-jardim, ruas macadamizadas ou } \\
\text { pavimentadas; }\end{array}$ & 0,25 a 0,50 \\
\hline $\begin{array}{l}\text { De subúrbios com alguma edificação: partes de arre- } \\
\text { baldes com pequena densidade de construções; }\end{array}$ & 0,10 a 0,25 \\
\hline $\begin{array}{l}\text { De matas, parques e campos de esporte: partes rurais, } \\
\text { áreas verdes, superfícies arborizadas, parques e cam- } \\
\text { pos de esporte sem pavimentação. }\end{array}$ & 0,05 a 0,20 \\
\hline
\end{tabular}
da bacia

Tabela 2 - Valores de C baseados nas características detalhadas das diversas superfícies presentes na bacia

\begin{tabular}{ll}
\hline Telhados perfeitos sem fuga; & 0,70 a 0,95 \\
\hline Superfícies asfaltadas em bom estado; & 0,85 a 0,90 \\
\hline $\begin{array}{l}\text { Pavimentação de paralelepípedos, ladrilhos ou blocos de } \\
\text { madeira com juntas bem tomadas; }\end{array}$ & 0,70 a 0,85 \\
\hline Para superfícies anteriores sem as juntas tomadas; & 0,50 a 0,70 \\
\hline Pavimentação de blocos inferiores sem as juntas tomadas; & 0,40 a 0,50 \\
\hline Estradas macadamizadas; & 0,25 a 0,60 \\
\hline Estradas e passeios de pedregulho; & 0,15 a 0,30 \\
\hline $\begin{array}{l}\text { Superfícies não-revestidas, pátios de estradas de ferro e } \\
\text { terrenos descampados, parques, jardins, dependendo da }\end{array}$ & 0,10 a 0,30 \\
\hline declividade; & 0,01 a 0,20
\end{tabular}

Fonte: Villela e Mattos (1980)

- área do lote: área total do lote $\left(\mathrm{m}^{2}\right)$;

- área de projeção das construções: corresponde às áreas edificadas nos lotes em projeção horizontal $\left(\mathrm{m}^{2}\right)$;

- taxa de ocupação (TO): relação entre a área construída em projeção horizontal e a área do lote (\%);

- taxa de ocupação e impermeabilização (TOI): relação entre a área construída em projeção horizontal acrescida da área impermeabilizada e a área do lote (\%), este parâmetro é referente à impermeabilização total existente no lote;

- porcentagem de área impermeabilizada diretamente conectada (AIDC);

- porcentagem de área impermeabilizada não-conectada (AINC);

- $\quad$ porcentagem de área permeável (AP).

\section{Estratificação}

Para realizar as análises e caracterizações pretendidas para Ribeirão Preto, foi necessário escolher um elemento espacial que estivesse presente em toda a cidade, que permitisse o cruzamento 
dos dados obtidos e proporcionasse uma estratificação de amostras, ou seja, a associação de elementos com mesmas características. Por essas razões, o elemento espacial escolhido foi o lote. A caracterização urbanística de Ribeirão Preto foi realizada a partir da análise de parâmetros relacionados ao uso e ocupação do solo nos lotes urbanos.

Buscando maior homogeneidade e especificidade desta caracterização, os lotes urbanos foram separados em estratos com o intuito de abrangê-los e agrupá-los com características semelhantes, levandose em consideração informações acerca dos lotes que poderiam ser conhecidas previamente. Portanto, a estratificação foi realizada de acordo com o uso e ocupação do solo.

Pode-se entender o estrato como um subconjunto de lotes que apresentam usos semelhantes ou, no caso de lotes residenciais, que tenham área compreendida em um determinado intervalo. Dessa forma, os lotes urbanos foram separados em 11 estratos, classificados da seguinte forma:

- estrato 1: formado por lotes institucionais, ou seja, ocupados por edificações ou áreas voltadas ao atendimento de interesses públicos, de capital público ou privado;

- estrato 2: lotes comerciais e industriais, ocupados por empresas de capital privado;

- estrato 3: lotes mistos e outros, ou seja, lotes ocupados com múltiplas utilizações ou que não se encaixam em outras classificações.

Os lotes residenciais foram re-estratificados segundo a área do lote e classificados da seguinte maneira:

- estrato 4: lotes residenciais com área menor que $125 \mathrm{~m}^{2}$;

- estrato 5: lotes residenciais com área entre 125 m² e 200 m²;

- estrato 6: lotes residenciais com área entre $200 \mathrm{~m}^{2}$ e $300 \mathrm{~m}^{2}$.

- estrato 7: lotes residenciais com área entre $300 \mathrm{~m}^{2}$ e $400 \mathrm{~m}^{2}$;

- estrato 8: lotes residenciais com área entre $400 \mathrm{~m}^{2}$ e $600 \mathrm{~m}^{2}$;

- estrato 9: lotes residenciais com área entre $600 \mathrm{~m}^{2}$ e 1200 m²;

- estrato 10: lotes residenciais com área superior a $1200 \mathrm{~m}^{2}$;

- estrato 11: lotes verticalizados, ou seja, ocupados por edifícios em altura superior a três pavimentos, independentemente do uso.

\section{Tamanho, proporcionalidade e aleatoriedade da amostragem}

Para definição do número de lotes que seriam analisados por meio das visitas de campo, foi realizado um estudo estatístico por amostragem estratificada aleatória e proporcional, conforme metodologia utilizada por Fontes e Barbassa (2003). Para tanto, necessitou-se de definição prévia da variável principal e o cálculo de sua variância, além do elemento morfológico a ser estudado. Como visto, o elemento morfológico escolhido foi o lote, e a variável principal utilizada para definir o número de amostras foi a taxa de ocupação (TO) por se tratar de um parâmetro urbanístico relacionado à área construída e à área livre do lote.

O tamanho da amostra, ou seja, o número de lotes a serem visitados foi determinado pela Equação 3, conforme Triola (1989):

$$
n=\frac{N * \sigma^{2}}{\sigma^{2}+(N-1) *\left(\frac{E}{z}\right)^{2}}
$$

onde:

$n$ : número de amostras total para coleta de campo;

$\sigma^{2}$ : variância da variável principal - taxa de ocupação (TO);

$N$ : tamanho da população - número total de lotes urbanos ocupados;

$E$ : erro máximo admitido na estimativa da média;

$z$ : quantil da distribuição normal correspondente a 96\% de confiabilidade na estimativa da média;

Utilizando os dados cadastrais da Prefeitura municipal de Ribeirão Preto (PMRP), foram calculados os valores da TO para todos os lotes e, então, observada a variância deste parâmetro, estimada em: $\sigma^{2}=(28,71)^{2}$.

A partir da Equação 3 foram estipulados: confiabilidade de 96\%, um erro máximo admitido na estimativa da média de 4\% (que está dentro da faixa costumeiramente aceita de 5\%), a variância estimada de $\sigma^{2}=28,71^{2}$ ), população de lotes ocupados $N=125.445$ (o tamanho da amostra foi definido em relação à população de lotes ocupados, pois estes foram os lotes visitados). Com esses dados, obteve-se uma amostra (n) de 198 lotes.

Para se obter a proporção da amostra relativa a cada um dos 11 estratos de lotes ocupados, foi utilizado o desvio padrão da TO referente a cada estrato por meio da Equação 4. A utilização desta ferramenta foi a que forneceu melhor distribuição e proporcionalidade entre as amostras dos estratos, além de distribuir teoricamente melhor as amostras, pois os estratos que apresentam maior variabilidade da TO terão maior número de amostras coletadas. Por esta razão, utilizou-se o desvio padrão da TO por estrato, $\sigma_{i}$, para cálculo do coeficiente de proporcionalidade, conforme Equação 4:

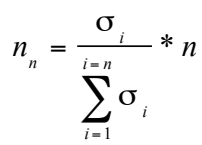

Equação 4

onde:

$n_{n}$ : número de amostras por estrato para coleta de campo;

$\sigma_{i}$ : desvio padrão da TO de cada estrato;

$\sum_{i=1}^{i=n} \sigma_{i}$ : somatório dos desvios padrões das TO's;

$n$ : número total de amostras para coleta de campo. 


\section{Estudo da impermeabilização e conectividade hidráulica do lote}

Através de visitas de campo, várias investigações foram realizadas, entre elas a quantificação da conectividade hidráulica dos lotes, ou seja, a quantidade de área presente no lote que drena as águas pluviais diretamente para o sistema de drenagem pública. Para levantar dados sobre a conectividade dos lotes, foram utilizadas as etapas descritas a seguir:

- visita de campo 1: durante a primeira visita de campo, foram quantificadas as áreas permeáveis e executou-se um croqui do lote com a disposição de áreas permeáveis, construídas e impermeabilizadas. Verificou-se também a presença de áreas impermeabilizadas não-conectadas (AINC).

- identificação dos lotes que apresentavam AINC e sua localização nos mapas e ortofotos;

- digitalização dos lotes que continham AINC, a partir das ortofotos;

- detalhamento dos telhados, com a finalidade de identificar o caminho percorrido pelas águas pluviais;

- visita de campo 2: foi realizada uma segunda visita somente aos lotes que possuíam AINC e que foram, por sua vez, verificadas e separadas das outras áreas impermeabilizadas;

- cálculo e quantificação das AINC, AIDC e AP.

A partir desta sequência, foram levantados os dados sobre conectividade hidráulica nos lotes. Ressalta-se a precisão dos dados coletados, pois foram medidos tanto através de ortofotos quanto por medições in loco.

Na Tabela 3, apresentam-se as características e valores médios analisados para cada estrato. Nas colunas 1 e 2 há a descrição do estrato e o número de amostras que foram coletadas para análise; na coluna 3 é apresentado o número de lotes; nas colunas 4 e 5 são apresentados, respectivamente, a área ocupada pelos lotes em $\mathrm{km}^{2}$ e a porcentagem de área representada por cada estrato com relação aos lotes ocupados no município. Nas colunas de 6 a 11 são apresentadas, respectivamente, as médias obtidas para área do lote, TO, TOI, AIDC, AINC e AP.

Através dos valores obtidos para TO, TOI, AIDC, AINC, AP, área de lote, algumas relações podem ser obtidas entre estes parâmetros, como a relação entre área do lote, área impermeabilizada diretamente conectada (AIDC), área impermeabilizada total, que é o mesmo que taxa de ocupação e impermeabilização (TOI), taxa de ocupação (TO); desta forma, através de dados conhecidos a respeito de lotes e obtidos a partir de informações em cadastros municipais, estimaram-se parâmetros de impermeabilização do solo nestes lotes.

\section{Estimativas de C}

Depois de os valores médios dos parâmetros sobre impermeabilização terem sido obtidos, para os diferentes estratos foram selecionadas e separadas seis sub-bacias, com o objetivo de estimar valores de C utilizando diferentes metodologias, inclusive adotando como valor de $C$ a média ponderada de área impermeabilizada diretamente conectada (AIDC). Utilizou-se o Método Racional para calcular as vazões e comparar as metodologias.

As sub-bacias foram escolhidas de acordo com a localização em relação ao centro da cidade e tipo de construções existente, conforme separação proposta na Tabela 1. A localização, formato e tamanho das sub-bacias estudadas encontram-se na Figura 1.

\section{Estimativa de $\mathrm{C}$ pelas características gerais de urbanização $\left(\mathrm{C}_{\text {URB }}\right)$}

O coeficiente de escoamento superficial para ocupações e usos variados em uma mesma bacia pode ser calculado usando-se a média ponderada, conforme Equação 5.

$C=\frac{C_{1} \cdot A_{1}+C_{2} \cdot A_{2}+\ldots .+C_{n} \cdot A_{n}}{A_{1}+A_{2}+\ldots .+A_{n}}$

Equação 5

Tabela 3 - Características gerais e valores médios da TO, TOI, AIDC, AINC, AP, área dos lotes para cada estrato

\begin{tabular}{|c|c|c|c|c|c|c|c|c|c|c|}
\hline \multirow{2}{*}{ Estrato } & \multirow{2}{*}{$\begin{array}{l}\text { Número de } \\
\text { amostras }\end{array}$} & \multirow{2}{*}{$\begin{array}{l}\text { Número } \\
\text { de lotes }\end{array}$} & \multirow{2}{*}{$\begin{array}{l}\text { Lotes ocupados } \\
\qquad\left(\mathrm{km}^{2}\right)\end{array}$} & \multirow{2}{*}{$\begin{array}{l}\text { \% área urbana por } \\
\text { lotes ocupados }\end{array}$} & \multirow{2}{*}{$\begin{array}{l}\text { Área média } \\
\text { por lote }\left(\mathrm{m}^{2}\right)\end{array}$} & \multicolumn{5}{|c|}{ Resultados médios por estrato (\%) } \\
\hline & & & & & & TO & TOI & AIDC & AINC & AP \\
\hline Col.1 & Col. 2 & Col. 3 & Col. 4 & Col. 5 & Col. 6 & Col. 7 & Col. 8 & Col. 9 & Col. 10 & Col. 11 \\
\hline 1- Institucional & 26 & 592 & 4,76 & 6 & 4105,5 & 54,3 & 79,8 & 74,7 & 5,1 & 20,2 \\
\hline 2- comercial/ industrial & 26 & 8732 & 13,28 & 17 & 816,7 & 67,3 & 85,6 & 76,8 & 8,8 & 14,4 \\
\hline 3- misto / outros & 20 & 7352 & 10,96 & 14 & 325,9 & 77,9 & 99,7 & 99,7 & 0 & 0,3 \\
\hline 4- res1: $\leq 125 \mathrm{~m}^{2}$ & 15 & 14082 & 1,39 & 2 & 102,5 & 77,4 & 98,7 & 98,1 & 0,6 & 1,3 \\
\hline 5- res2: $126-200 \mathrm{~m}^{2}$ & 18 & 43065 & 7,53 & 9 & 173,1 & 54,3 & 90,4 & 79,8 & 10,6 & 9,6 \\
\hline 6- res3: $201-300 \mathrm{~m}^{2}$ & 17 & 28990 & 7,25 & 9 & 249,6 & 56,8 & 95,5 & 89,8 & 4,8 & 4,5 \\
\hline 7- res4: $301-400 m^{2}$ & 16 & 8707 & 3,01 & 4 & 341,6 & 48,8 & 80,7 & 69,2 & 11,5 & 19,3 \\
\hline 8- res5: $401-600 \mathrm{~m}^{2}$ & 15 & 6145 & 2,93 & 4 & 458,7 & 49,5 & 81,7 & 73,5 & 8,2 & 18,3 \\
\hline 9- res6: 601 - $1200 \mathrm{~m}^{2}$ & 15 & 2270 & 1,84 & 2 & 812,9 & 41,2 & 73,4 & 64,1 & 9,3 & 26,6 \\
\hline 10- res7: $1200-2000 \mathrm{~m}^{2}$ & \multirow{2}{*}{10} & \multirow{2}{*}{2416} & 1,18 & 1,5 & 1459,7 & 48,5 & 70,3 & 66,9 & 3,5 & 29,6 \\
\hline 10- res7: > $2000 \mathrm{~m}^{2}$ & & & 15,68 & 19,5 & 7596,9 & 3,5 & 4,5 & 0 & 4,5 & 95,5 \\
\hline 11- verticalizados & 20 & 3094 & 10,02 & 13 & 1275,6 & 54,6 & 93 & 92,5 & 0,5 & 7 \\
\hline Total & 198 & 125445 & 79,85 & 100 & - & - & - & - & - & - \\
\hline
\end{tabular}


onde:

$C$ : coeficiente de escoamento superficial médio calculado para a bacia;

$C_{n}$ : coeficiente de escoamento superficial de área específica dentro da bacia;

$A_{n}$ : área $\left(\mathrm{km}^{2}\right)$ relativa ao coeficiente $\mathrm{C}_{\mathrm{n}}$.

Neste caso, cada área $\mathrm{A}_{\mathrm{i}}$ apresenta características de urbanização homogêneas em relação ao uso, à sua posição em relação ao centro da cidade, à densidade de edificações, à presença de superfícies livres, etc. $\mathrm{O} \mathrm{C}_{\mathrm{i}}$ correspondente à área $\mathrm{A}_{\mathrm{i}}$ pode ser obtido de tabelas de diversas referências como Tucci, Porto e Barros (1995), Villela e Mattos (1980), conforme Tabela 1, extraída de Wilken (1978).

Adotaram-se para cálculo de C o valor médio, o máximo e o mínimo, representando a faixa de variação dada na Tabela 1 para cada tipo de zona ou área $\mathrm{A}_{\mathrm{i}}$, neste caso tomando cada uma das seis subbacias como uma área $A_{i}$.

\section{Estimativa de $\mathrm{C}$ pelas características detalhadas da superfície $\left(C_{D E T}\right)$}

Utilizando-se a Equação 5, calculou-se o coeficiente de escoamento, diferenciando-se as estimativas de $A_{i}$ e de $C_{i}$. Cada área $A_{i}$ da bacia refere-se a um mesmo tipo de superfície, como telhados, vias macadamizadas, apedregulhadas, não-pavimentadas, quintais, etc. $\mathrm{O}$ $\mathrm{C}_{\mathrm{i}}$ pode ser obtido de tabelas com tipos de superfícies detalhadas, encontradas em vários livros de hidrologia e drenagem urbana, conforme Tabela 2, extraída de Villela e Mattos (1980).

As áreas A’s podem ser estimadas por diversos métodos. Neste item, as áreas $A_{i}$ 's e seus respectivos $C_{i}$ 's e o $C_{D E T}$, calculados pela Equação 5, foram estimados como mostra a Tabela 4, tomando-se a sub-bacia do córrego do Tanquinho (SBHT1), como exemplo:

a) obtiveram-se as áreas e usos de todos os lotes junto ao cadastro da Prefeitura Municipal;

b) os lotes foram separados em áreas homogêneas (estratos), representativos das áreas A's, conforme coluna 1 da Tabela 4;

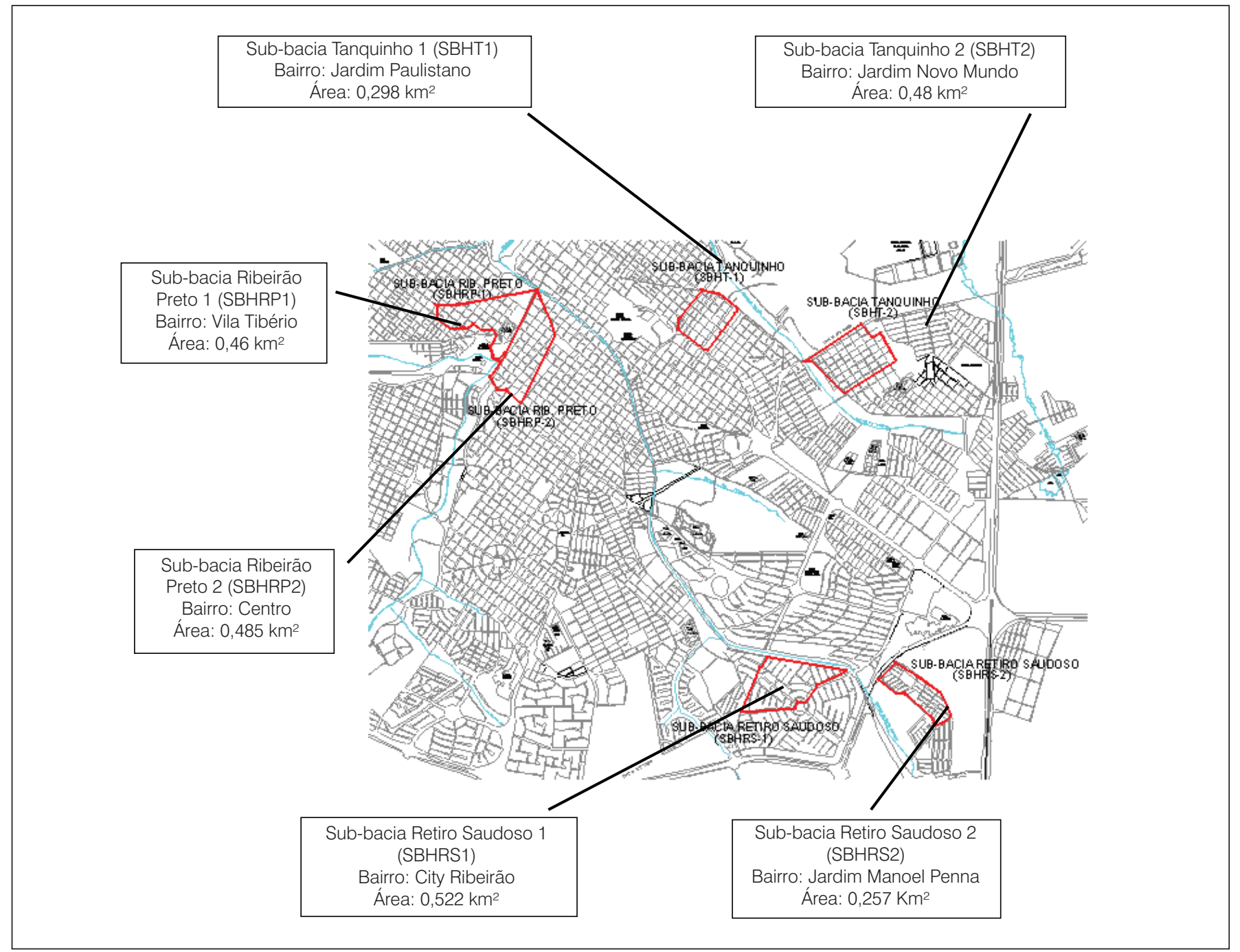

Figura 1 - Localização das sub-bacias estudadas em Ribeirão Preto (SP) 
c) calcularam-se as áreas totais dos lotes A's conforme coluna 2;

d) os percentuais das colunas 3, 5, 7 e 9 foram obtidos estatisticamente para Ribeirão Preto, conforme resultados apresentados na Tabela 3;

e) adotaram-se valores de C, expressos nas colunas 4, 6, 8, 10, baseados nos valores apresentados na Tabela 2, de acordo com a superfície detalhada;

f) a coluna 11 apresenta os valores de $C$ ponderados por área $A_{i}$;

g) $\mathrm{O}_{\mathrm{DET}}$ para a sub-bacia do Tanquinho foi obtido calculando-se a média ponderada dos $C_{i}$ da coluna 11 e a área $A_{i}$ da coluna 2, resultando em $C_{D E T}$ médio igual a 0,66.

$\mathrm{Na}$ Tabela 4 apresenta-se apenas o valor médio de $\mathrm{C}$ para as diferentes características de $A_{i}$, mas também foram usados valores máximos e mínimos de C.

\section{Estimativa de $\mathrm{C}_{\text {AIDC }}$ pelas áreas impermeabilizadas diretamente conectadas do lotes}

Outro método para estimar o coeficiente de escoamento superficial é adotar este valor como a porcentagem de AIDC, presente na bacia, bairro ou área urbana analisada, conforme Equação 6. Este procedimento corresponde à versão original britânica do Método Racional do começo do século 20.

$Q=C_{A I D C} * I * A=A I D C * I$

Equação 6 onde:

$Q$ : vazão $\left(\mathrm{m}^{3} / \mathrm{s}\right)$;

$C_{A I D C}$ : coeficiente de escoamento superficial correspondente à porcentagem de área impermeável diretamente conectada;

I: intensidade pluviométrica $(\mathrm{mm} / \mathrm{h})$;

$A$ : área da bacia $\left(\mathrm{km}^{2}\right)$;

AIDC: área $\left(\mathrm{km}^{2}\right)$ correspondente ao total impermeabilizado diretamente conectado.

Na Equação 6, "A" é a área total da bacia, AIDC a área impermeável diretamente conectada da bacia expressa em $\mathrm{m}^{2}, \mathrm{C}_{\mathrm{AIDC}}$ o coeficiente de escoamento superficial e I a intensidade da chuva.

Apresentam-se na Tabela 3 os valores percentuais de AIDC obtidos para cada estrato em Ribeirão Preto (SP). Nesta tabela, são observados também os valores percentuais de AINC e AP.

Neste item, $\mathrm{C}_{\mathrm{AIDC}}$ foi estimado como mostra a Tabela 5, tomandose como exemplo, a mesma sub-bacia do Tanquinho (SBHT1). Os passos a, b, c são idênticos aos do cálculo de $C_{D E T}$ :

d) mostra-se na coluna 4 o percentual de área impermeabilizada diretamente conectada (AIDC), calculado estatisticamente para Ribeirão Preto;

e) o $C_{\text {AIDC }}$ para a sub-bacia do Tanquinho foi calculado como a média ponderada de percentual de AIDC da coluna 4 e a área $A_{i}$ da coluna 2, dividido pela área total e por 100 , resultando em $C_{\text {AIDC }}$ igual a 0,795 . Observa-se que este $\mathrm{C}_{\mathrm{AIDC}}$ considera $100 \%$ de escoamento das áreas impermeabilizadas diretamente conectadas,

Tabela 4 - Exemplo de estimativa do $C_{D E T}$ para sub-bacia do córrego do Tanquinho em Ribeirão Preto (SP)

\begin{tabular}{|c|c|c|c|c|c|c|c|c|c|c|}
\hline \multirow[t]{2}{*}{ Tipos de áreas $\mathrm{Ai}$} & \multirow[t]{2}{*}{ Área $\left(\mathrm{m}^{2}\right)$} & \multicolumn{2}{|c|}{$\begin{array}{l}\text { Área construída } \\
\text { diretamente } \\
\text { conectada }\end{array}$} & \multicolumn{2}{|c|}{$\begin{array}{c}\text { Área livre } \\
\text { impermeabilizada } \\
\text { diretamente } \\
\text { conectada }\end{array}$} & \multicolumn{2}{|c|}{$\begin{array}{c}\text { Área } \\
\text { impermeabilizada } \\
\text { não-conectada }\end{array}$} & \multicolumn{2}{|c|}{$\begin{array}{l}\text { Área livre } \\
\text { permeável }\end{array}$} & \multirow[t]{2}{*}{$C$ ponderado por $A_{i}$} \\
\hline & & (\%) & $\mathrm{C}_{\text {adot }}{ }^{1}$ & $(\%)$ & $\mathrm{C}_{\text {adot }}{ }^{2}$ & (\%) & $\mathrm{C}_{\text {adot }^{3}}{ }^{3}$ & $(\%)$ & $\mathrm{C}_{\text {adota }}{ }^{4}$ & \\
\hline Col. 1 & Col. 2 & Col. 3 & Col. 4 & Col. 5 & Col. 6 & Col. 7 & Col. 8 & Col. 9 & Col. 10 & Col. 11 \\
\hline A1-Institucional & 73.151 & 54,34 & 0,825 & 20,4 & 0,775 & 5,08 & 0,2 & 20,18 & 0,1 & 0,64 \\
\hline A2-Comercio/indústria & 8.889 & 67,26 & 0,825 & 9,53 & 0,775 & 8,84 & 0,2 & 14,38 & 0,1 & 0,66 \\
\hline A3-Misto/outros & 9.347 & 77,97 & 0,825 & 21,71 & 0,775 & 0 & 0,2 & 0,32 & 0,1 & 0,81 \\
\hline A4-Residencial $<125$ & 2.072 & 77,45 & 0,825 & 20,63 & 0,775 & 0,58 & 0,2 & 1,33 & 0,1 & 0,8 \\
\hline A5-Residencial 125-200 & 12.701 & 54,34 & 0,825 & 25,44 & 0,775 & 10,58 & 0,2 & 9,64 & 0,1 & 0,68 \\
\hline A6-Residencial 200-300 & 63.689 & 56,76 & 0,825 & 33,92 & 0,775 & 4,8 & 0,2 & 4,51 & 0,1 & 0,75 \\
\hline A7-Residencial 300-400 & 16.387 & 48,82 & 0,825 & 20,4 & 0,775 & 11,46 & 0,2 & 19,32 & 0,1 & 0,6 \\
\hline A8-Residencial 400-600 & 15.630 & 49,53 & 0,825 & 23,94 & 0,775 & 8,21 & 0,2 & 18,32 & 0,1 & 0,63 \\
\hline A9-Residencial 600-1200 & 5.239 & 41,19 & 0,825 & 22,89 & 0,775 & 9,33 & 0,2 & 26,59 & 0,1 & 0,56 \\
\hline A10-Residencial > 1200 & 4.778 & 48,55 & 0,825 & 18,33 & 0,775 & 3,47 & 0,2 & 29,65 & 0,1 & 0,58 \\
\hline A11-Verticalizado & 10.146 & 54,64 & 0,825 & 37,83 & 0,775 & 0,53 & 0,2 & 7,01 & 0,1 & 0,75 \\
\hline A12-Vagos & 5.523 & 0 & 0,825 & 0 & 0,775 & 0 & 0,2 & 100 & 0,1 & 0,1 \\
\hline A13-Áreas verdes & 13.193 & 0 & 0,825 & 0 & 0,775 & 0 & 0,2 & 100 & 0,1 & 0,1 \\
\hline A14-Rua/calçada & 58.007 & 0 & 0,825 & 100 & 0,775 & 0 & 0,2 & 0 & 0,1 & 0,78 \\
\hline Total & 298.752 & & & & & & ET para & ub-bacia & & 0,66 \\
\hline
\end{tabular}

'Corresponde a 'telhados perfeitos sem fuga' segundo Tabela 2 ;

${ }^{2}$ Corresponde a 'pavimentação de paralelepípedos, ladrilhos ou blocos de madeira com juntas bem tomadas' segundo Tabela 2;

${ }^{3}$ Corresponde a 'superfícies não-revestidas, pátios de estradas de ferro e terrenos descampados, parques, jardins, dependendo da declividade' segundo Tabela 2;

${ }^{4}$ Corresponde a "solo na natureza e do subsolo' segundo Tabela 2. 
desprezando as contribuições tanto das áreas impermeabilizadas não-conectadas (AINC) quanto das áreas permeáveis (AP).

\section{Análises das estimativas de C}

Os valores mínimos, médios e máximos referentes aos coeficientes de escoamento superficial $C_{\mathrm{URB}}, \mathrm{C}_{\mathrm{DET}}, \mathrm{C}_{\mathrm{AIDC}}$ para as seis sub-bacias estão apresentados na Tabela 6 . As diferenças entre os valores obtidos podem ser explicadas tanto pela definição das áreas homogêneas $A_{1}$ 's, quanto pela adoção dos respectivos C's.

No cálculo de $\mathrm{C}_{\mathrm{URB}}$, as áreas $\mathrm{A}_{\mathrm{i}}$ 's foram definidas de acordo com características gerais de urbanização da sub-bacia, conforme Tabela 1. Desta forma, as dificuldades em enquadrar a urbanização nas descrições da Tabela 1 estão em saber quando começa uma área e termina outra, o que pode ocasionar erros no computo das áreas homogêneas A.s. Além disso, as características de urbanização descritas na Tabela 1, geralmente, são limitadas e vagas ao representar os parcelamentos, usos e ocupações das condições brasileiras.

No cálculo de $C_{D E T}$ e $C_{\text {AIDC }}$ as áreas homogêneas $A_{1}$ 's são estimadas a partir de percentuais de áreas construídas, impermeabilizadas diretamente conectadas, impermeabilizadas não-conectadas e permeáveis, obtidos

Tabela 5 - Exemplo de cálculo de $\mathrm{C}_{\text {AIDC }}$ ponderado para a sub-bacia do córrego do Tanquinho em Ribeirão Preto (SP) (SBHT1)

\begin{tabular}{llll}
\hline Tipos de áreas Ai (Estratos) & Áreas Ais $\left(\mathrm{m}^{2}\right)$ & Áreas Ais (\%) & AIDC (\%) \\
\hline Col. 1 & Col. 2 & Col. 3 & Col. 4 \\
\hline A1-Institucional & 73.151 & 24,5 & 74,7 \\
\hline A2-Comércio/indústria & 8.889 & 3 & 76,8 \\
\hline A3-Misto/outros & 9.347 & 3,1 & 99,7 \\
\hline A4-Res.<125 & 2.072 & 0,7 & 98,1 \\
\hline A5-Res.125-200 & 12.701 & 4,3 & 79,8 \\
\hline A6-Res.200-300 & 63.689 & 21,3 & 90,7 \\
\hline A7-Res.300-400 & 16.387 & 5,5 & 69,2 \\
\hline A8-Res.400-600 & 15.630 & 5,2 & 73,5 \\
\hline A9-Res.600-1200 & 5.239 & 1,8 & 64,1 \\
\hline A10-Res.>1200 & 4.778 & 1,6 & 66,9 \\
\hline A11-Verticalizados & 10.146 & 3,4 & 92,5 \\
\hline A12-Vagos & 5.523 & 1,8 & 0 \\
\hline A13-Áreas verdes & 13.193 & 4,4 & 0 \\
\hline A14-Rua/calçadas & 58.007 & 19,4 & 100 \\
\hline Total & 298.752 & 100 & \\
Caidc ponderado para a sub-bacia SBHT1 & & 0,795
\end{tabular}

estatisticamente de medidas in loco. Com isto, aumenta-se a fidelidade na descrição e mensuração destas áreas, inclusive considerando-se áreas nãovisíveis em fotos aéreas. Esse procedimento acaba por elevar a quantidade das áreas impermeabilizadas, o que pode explicar a razão de os valores de $C_{\text {AIDC }}$ e $C_{\text {DET }}$ serem geralmente superiores ao $C_{\mathrm{URB}}$, conforme Tabela 6. Os coeficientes $\mathrm{C}_{i}$ 's, também adotados a partir do detalhamento das superfícies, levam a um comportamento hidrológico esperado para estas áreas.

Portanto, admite-se que as AIDC's tenham um volume escoado próximo do volume precipitado, pois o escoamento é direcionado ao sistema de drenagem, sem oportunidade de infiltração. Desta forma, admite-se que o coeficiente de escoamento superficial possa ser tomado igual ao percentual de áreas conectadas, negligenciandose as pequenas contribuições das áreas permeáveis. Mesmo assim, observa-se, na Tabela 6, que em 5 das 6 áreas estudadas o valor de $C_{\text {AIDC }}$ supera o valor de $C_{D E T}$ em seu limite máximo em até $16 \%$ para SBHRP1, à exceção de uma sub-bacia, onde a quantidade de AIDC é baixa, fazendo com que as áreas permeáveis passem a contribuir de forma mais importante para a geração do escoamento.

\section{Vazão de pico de sub-bacias para diferentes valores de "C"}

Utilizando-se o Método Racional, foi calculada a vazão de pico para os hidrogramas das sub-bacias separadas, com coeficientes de escoamento superficial iguais aos valores médios de $C_{U R B}, C_{D E T}$, e $\mathrm{C}_{\mathrm{AIDC}}$. Os hidrogramas estão apresentados nas Figuras 2 a 7.

Observa-se, nos hidrogramas, que os maiores picos ocorrem quando utilizado como coeficiente de escoamento o $\mathrm{C}_{\mathrm{AIDC}}$, podendo atingir um pico de vazão duas vezes maior do que o calculado com o $\mathrm{C}_{\text {URB }}$, conforme Figura 7. Quando a quantidade de áreas impermeáveis diretamente conectadas é baixa, o pico de vazão referente ao cálculo com o $\mathrm{C}_{\mathrm{DET}}$ médio se iguala ao calculado com o $\mathrm{C}_{\mathrm{AIDC}}$, conforme Figura 6. Em áreas centrais, o pico de vazão calculado com o $\mathrm{C}_{\mathrm{URB}}$ se iguala ao calculado com o $C_{\text {AIDC }}$, como se observa na Figura 5.

\section{Conclusões e recomendações}

Neste estudo, a análise da impermeabilização do solo em Ribeirão Preto apresentou-se elevada para os lotes ocupados, onde

Tabela 6 - Coeficientes de escoamento superficial calculados pelos três métodos para as seis sub-bacias em Ribeirão Preto (SP)

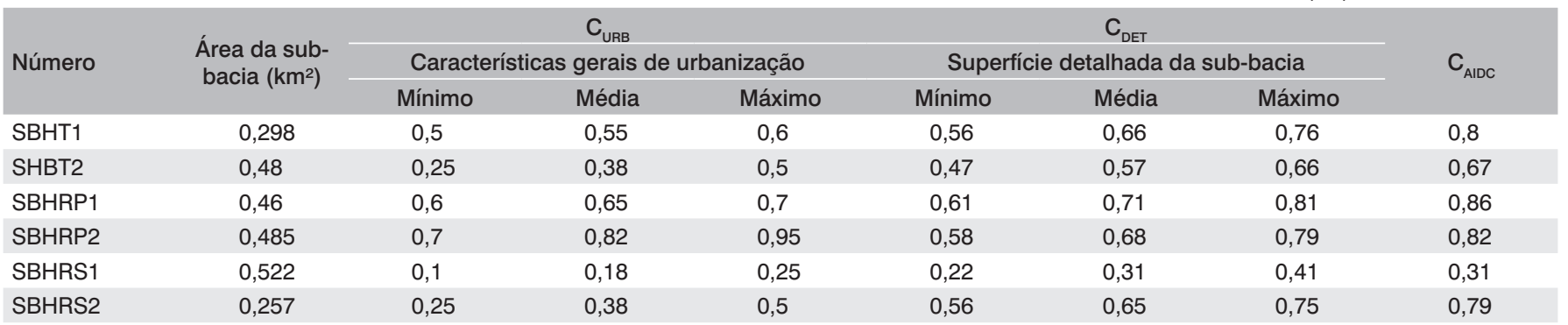




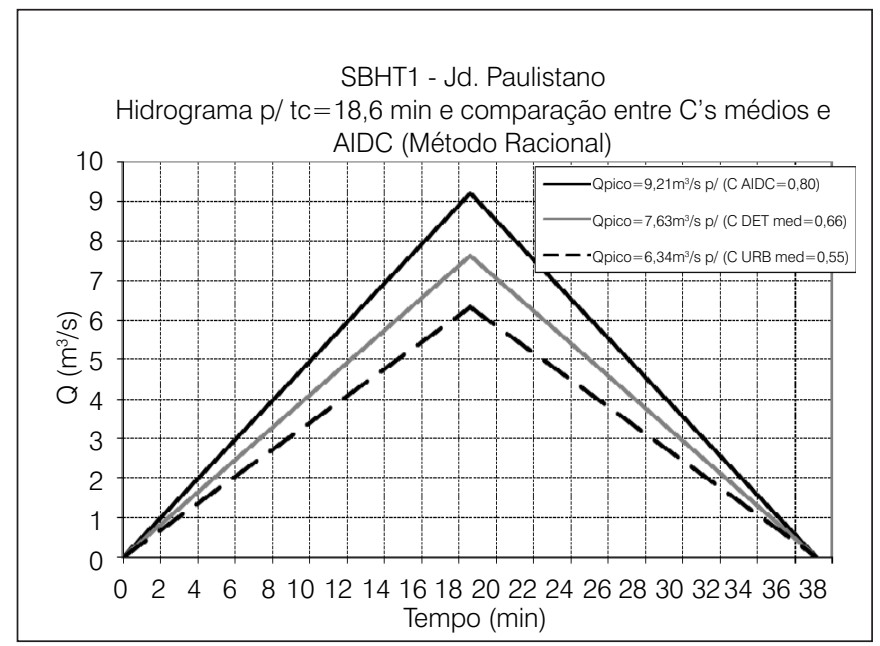

Figura 2 - Hidrograma na saída da SBHT1 obtido com Método Racional (Tr) 10 anos

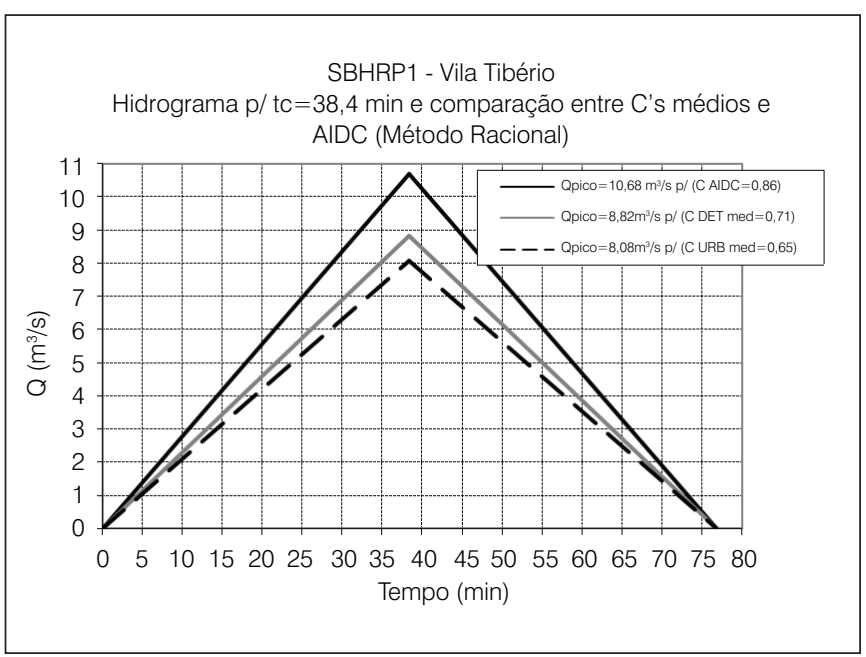

Figura 4 - Hidrograma saída da SBHRP1 obtido com Método Racional (Tr) 10 anos

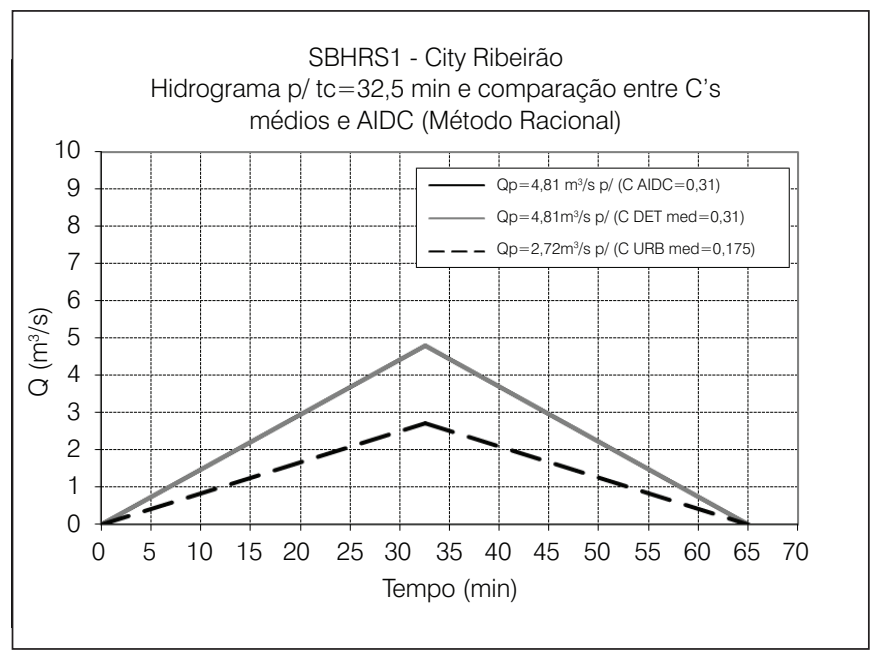

Figura 6 - Hidrograma saída da SBHRS1 obtido com Método Racional (Tr) 10 anos

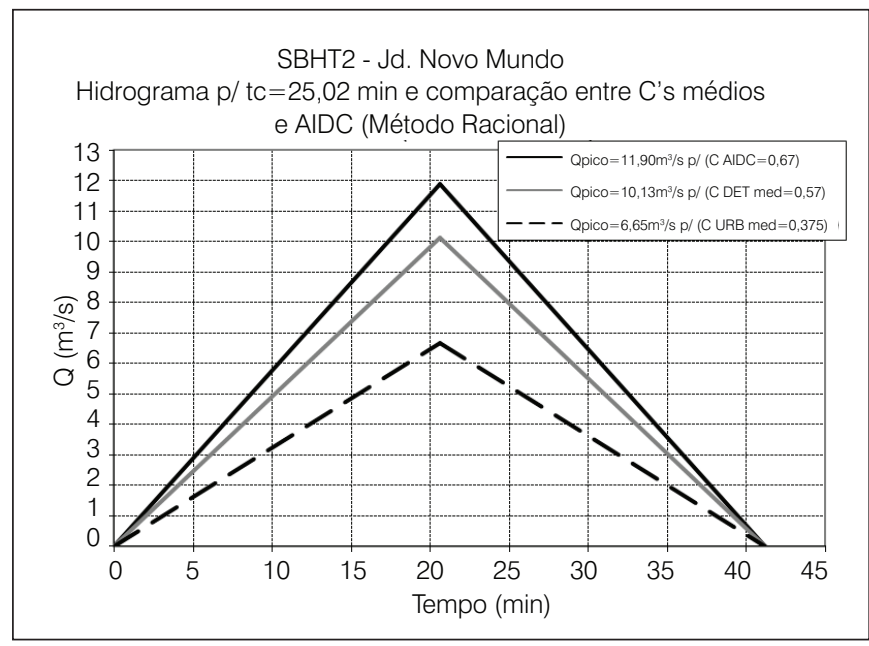

Figura 3 - Hidrograma na saída da SBHT2 obtido com Método Racional (Tr) 10 anos

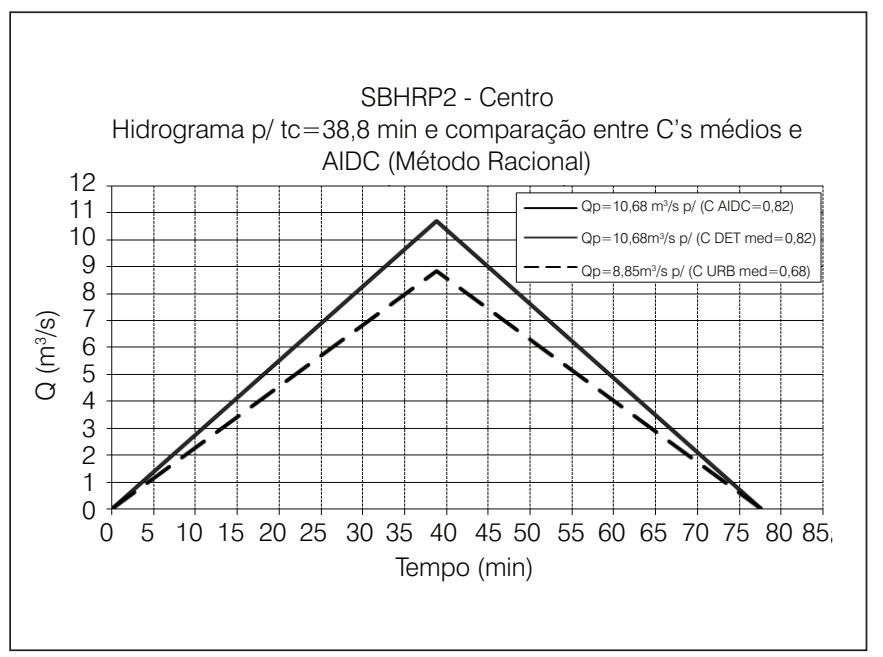

Figura 5 - Hidrograma saída da SBHRP2 obtido com Método Racional (Tr) 10 anos

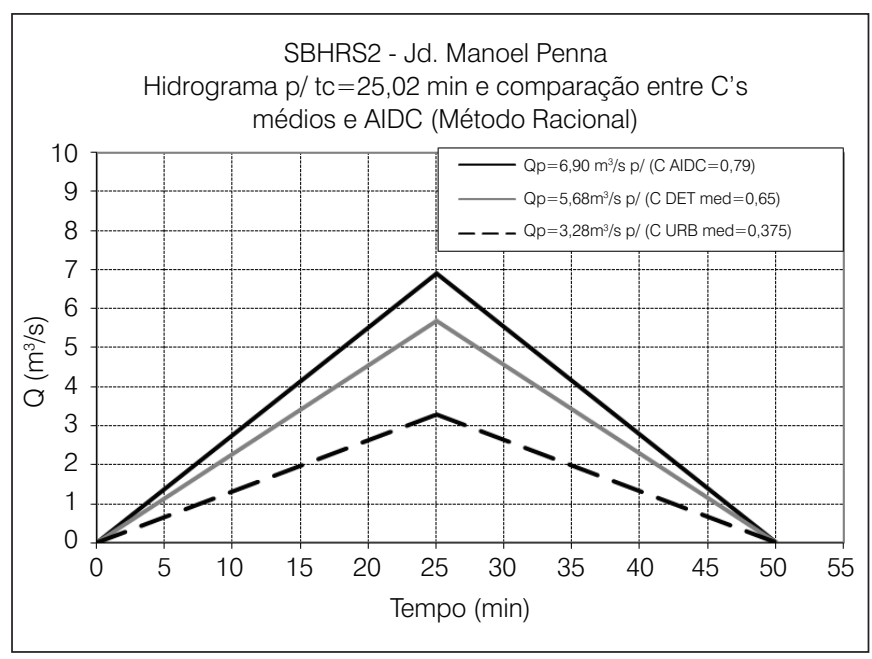

Figura 7 - Hidrograma saída da SBHRS2 obtido com Método Racional (Tr) 10 anos 
a menor taxa de impermeabilização foi superior a 70\% da área do lote. Observou-se também que quanto menor a área do lote, maior é a porcentagem impermeabilizada do lote.

Estimou-se, ainda, o coeficiente de escoamento superficial em seis sub-bacias, através de três métodos que apresentaram valores diferenciados devido à determinação das áreas homogêneas A 's, e à adoção dos coeficientes de escoamento das respectivas áreas A's.

A metodologia utilizada na representação de áreas homogêneas, com estratificação de lotes, a partir do tamanho e uso de solo, representou de forma confiável a urbanização esperada para a cidade de Ribeirão Preto e para as sub-bacias, pois este levantamento foi realizado por estudo estatístico e visitas in loco. Por outro lado, a descrição urbana da Tabela 1 não correspondeu como o esperado às condições de urbanização dos objetos estudados, prejudicando os valores estimados para $C_{U R B}$. Portanto, a utilização de $C_{U R B}$ deve ser feita com muita reserva.

A estimativa de $\mathrm{C}$ que representou mais detalhadamente as características da bacia foi o $C_{D E T}$, pois no seu cálculo foram levadas em conta todas as áreas que sabidamente contribuem para o escoamento, inclusive aquelas que têm potencial para infiltração. Recomenda-se que o $C_{D E T}$ seja calculado empregando-se os $C_{i}$ 's em seu limite superior indicado na literatura.

O coeficiente de escoamento superficial estimado de acordo com o percentual de AIDC, ou seja, $C_{\text {AIDC }}$, e consequentemente os picos de vazão calculados com estes valores, apresentaram-se superiores aos encontrados quando da utilização do $C_{D E T}$, conforme comparação entre as colunas 8 e 9 da Tabela 6, e também os hidrogramas apresentados nas Figuras 2, 3, 4, 5 e 7. Dessa forma, recomenda-se a utilização de $C_{\text {AIDC }}$ como coeficiente de escoamento superficial devido à facilidade em sua utilização e determinação de seus valores, utilizando-se parâmetros urbanísticos conhecidos e de fácil disponibilidade em cadastros municipais.

Espera-se, com este trabalho, contribuir para um melhor entendimento da ocupação, impermeabilização do solo e suas características, principalmente no que diz respeito à AIDC e à possibilidade de utilização deste parâmetro como coeficiente de escoamento superficial para uso do método racional.

Ressalta-se, ainda, que a quantificação da impermeabilização urbana é útil para outros métodos de previsão de enchentes e muito importante como parâmetro urbanístico.

Considera-se a estimativa dos percentuais de AIDC, AINC e AP própria para as cidades, pois estes procedimentos permitem conhecer detalhadamente a superfície urbana, com grande confiabilidade nos resultados, uma vez que esta metodologia já está definida. Caso não haja possibilidade de levantamento desses parâmetros e a cidade tenha as características de urbanização de Ribeirão Preto, sugere-se usar os valores desta cidade.

\section{Referências}

BOOTH, D.B.; JACKSON, C.R. Urbanization of aquatic systems: degradation thresholds, stormwater detection, and limits of mitigation. Journal of the American Water Resources Association, v. 22, n. 5, p. $1077-$ 1089, 1997.

BOYD, M.J.; BUFILL, M.C.; KNEE, R.M. Predicting pervious and impervious storm runoff from urban drainage basins. Hydrological Sciences Journal, v. 39, n. 4, p. 321-332, 1994.

. Pervious and impervious runoff in urban catchments. Hydrological Sciences Journal, v. 38, n. 6, p. 463-478, 1993.

FONTES, A.R.M.; BARBASSA, A.P. Diagnóstico e prognóstico da ocupação e impermeabilização urbanas. Revista Brasileira de Recursos Hídricos. Porto Alegre (RS), v. 8, n. 2, p. 137-147, 2003

Análise de parâmetros urbanísticos de drenagem pluvial. In: XIV SIMPÓSIO BRASILEIRO DE RECURSOS HÍDRICOS, Aracaju (SE), 2000. Anais Eletrônicos. Porto Alegre: ABRH, 1 CD, 2001.

GAROTTI, L.M.; BARBASSA, A.P. Impermeabilização e conectividade de lotes da cidade de Ribeirão Preto - SP. In: XVI SIMPÓSIO BRASILEIRO DE RECURSOS HÍDRICOS, João Pessoa, Anais em CD-ROM, 2005.

GAROTTI, L.M.; IMOTO, T.S.; BARBASSA, A.P. Uso da área impermeabilidade diretamente conectada como coeficiente de escoamento superficial e comparação com outras estimativas. In: VII
ENCONTRO NACIONAL DE ÁGUAS URBANAS. Anais... São Carlos. v. 1. CD-ROM, 2007.

LEE, J.G.; HEANEY, J.P. Estimation of urban imperviousness and its impacts on stormwater systems. Journal of Water Resources Planning and Management, v. 39, n. 4, p. 321-332, 2003.

SECRETARIA MUNICIPAL DE PLANEJAMENTO URBANO DE RIBEIRÃO PRETO. Cadastro Técnico Municipal: dados cadastrais. Ribeirão Preto, 2004a.

SECRETARIA MUNICIPAL DE PLANEJAMENTO URBANO DE RIBEIRÃO PRETO. Mapas do município (uso do solo, base de referencias, hidrografia). Ribeirão Preto, 2004b.

TRIOLA, M.F. Elementary statistics. 4 ed. New York: The Benjamin Cummings Publishing Company Inc., 1989.

TUCCI, C.E.M.; PORTO, R.L.; BARROS, M.T. (Org.) Drenagem urbana. 1. ed. Porto Alegre, RS: Universidade, 2005. v. 5. Coleção ABRH de Recursos Hídricos.

VILLELA, S.M.; MATTOS, A. Hidrologia aplicada. 2 ed. São Paulo: McGraw-Hill do Brasil Ltda., 1980.

WILKEN, P.S. Engenharia de drenagem superficial. 1 ed. São Paulo: CETESB, 1978. 